bioRxiv preprint doi: https://doi.org/10.1101/2022.02.20.481216; this version posted February 20, 2022. The copyright holder for this

\title{
Active muscular hydraulics
}

\author{
Suraj Shankar ${ }^{1}$, L. Mahadevan ${ }^{1,2,3 *}$ \\ ${ }^{1}$ Department of Physics, Harvard University, Cambridge, MA 02138, USA \\ ${ }^{2}$ Paulson School of Engineering and Applied Sciences, Harvard University, Cambridge, MA 02138, USA \\ ${ }^{3}$ Department of Organismic and Evolutionary Biology, Harvard University, Cambridge, MA 02138, USA \\ ${ }^{*}$ Correspondence to: lmahadev@g.harvard.edu
}

(Dated: February 20, 2022)

\begin{abstract}
What limits the rate of contraction in muscle? The molecular cycling of actomyosin crossbridges has typically been assumed to be rate limiting, but muscle is a soft, wet and active tissue that is spatially heterogeneous and heirarchically organized across multiple scales. We propose a minimal multiscale model that describes muscle as an active sponge and show how contractions generically induce intracellular fluid flow and power active hydraulic oscillations which dictate the fastest rate of muscle contraction. A key feature is the presence of spatially heterogeneous strains and local volumetric deformations, which we validate by reanalyzing data from existing experiments across different species and muscle types. We further demonstrate that the viscoelastic response of muscle is naturally nonreciprocal (or 'odd') due to its active and anisotropic nature and highlight a novel consequence, whereby periodic cycles in strain alone can produce work. Our work suggests a revised view of muscle dynamics that emphasizes the multiscale spatial aspects in addition to temporal aspects, with implications for physiology, biomechanics and locomotion.
\end{abstract}

Muscle is the primary driver of nearly all motion across the animal kingdom, yet the biophysical limits of muscle's performance remain poorly explained. Nowhere is this more apparent than in the spectacular behavior powered by ultrafast contractions of muscle, such as in high frequency sound production by rattlesnakes, fish swimbladders and songbirds, or in insect flight, where operating frequencies can range between $10^{2}-10^{3} \mathrm{~Hz}$ [1, 2]. The underlying rapid millisecond contractions of muscle pose a natural question - what determines the fastest rate at which muscle can contract? Since the pioneering work by H. E. Huxley, A. F. Huxley and others reviewed in [3, 4], much work has focused on elucidating the biochemical details of the rate-limiting steps at the molecular level, i.e., the binding kinetics of actomyosin crossbridges [57]. But muscle is a complex structured tissue that is heirarchically organized across multiple spatiotemporal scales [8] (Fig. 1A), suggesting that molecular kinetics may not be the sole limiter of contraction speed. On the mesoscale, striated muscle cells are soft, wet and active materials composed of a dense, anisotropic and actively contracting polymeric lattice (sarcomeres forming the myofibril), bathed in cytosol.

This coarse-grained view of muscle suggests a new hypothesis: muscle behaves as an active fluid-filled sponge (Fig. 1B). Upon activation, intact muscle fibers cannot contract everywhere homogeneously (due to global incompressibility in the presence of an intact sarcolemma), but it can do so locally by slowly squeezing fluid through the pores of the myofilament lattice, a process that is potentially rate-limiting. That water movement may play a role in muscle contraction has been noted in old studies $[9,10]$, including in early experiments by Szent-Györgi $[11,12]$ on extracted actomyosin threads that expel water as they violently contract (Fig. 1C). More subtly, ac- tive crossbridges are also known to produce transverse (radial) forces in addition to longitudinal ones, that can change the local volume of the sarcomere [13, 14]. And finally, spatially nonuniform strains (Fig. 1D) have been reported during tetanic contractions of intact muscle fibres, both ex-vivo $[15,16]$ and in-vivo [17, 18], suggesting that local strain gradients and the accompanying pressure gradients and fluid hydraulics may all be relevant dynamically.

Recent experiments probing the rapid dynamics of the myofilament lattice through sophisticated microscopy and small-angle X-ray scattering techniques [19-25] now allow for a quantification of some of these observations. We reanalyzed data on active oscillations of muscle fibers from various experiments performed across different muscle types and species to obtain time traces of local transverse $\left(\epsilon_{\perp}\right)$ and longitudinal $\left(\epsilon_{z z}\right)$ strains in the sarcomere (Fig. 2, see SI for details). Spontaneous contractile oscillations with frequency $\omega \sim 1 \mathrm{~Hz}$ can emerge in glycerinated skeletal muscle fibers (rabbit psoas, Fig. 2A-B) $[19,20]$, with periodic strain deformations that deviate significantly from being locally isochoric, i.e., they are not volume preserving (Fig. 2, black line). In-vivo measurements of the sarcomere geometry in asynchronous flight muscle of Drosophila [21-23] shows that, remarkably, the lattice contracts with a constant lattice spacing [22], hence $\epsilon_{\perp} \approx 0$ under natural flight conditions (wingbeat frequency $\omega \approx 156 \mathrm{~Hz}$; Fig. $2 \mathrm{C}$ ). On the other hand, synchronous flight muscle of Manduca sexta displays periodic lattice dilations and contractions under physiological conditions ( $\omega \approx 25 \mathrm{~Hz}$; Fig. 2D), both in-vivo [25] and ex-vivo [24]. Importantly, in all of the above examples, deformations of the myofilament lattice fail to preserve its local volume $\left(\epsilon_{z z}+\epsilon_{\perp} \neq 0\right)$, thereby necessitating fluid movement through the sarcomere and thus lead to 
bioRxiv preprint doi: https://doi.org/10.1101/2022.02.20.481216; this version posted February 20, 2022. The copyright holder for this preprint (which was not certified by peer review) is the author/funder. All rights reserved. No reuse allowed without permission.

A

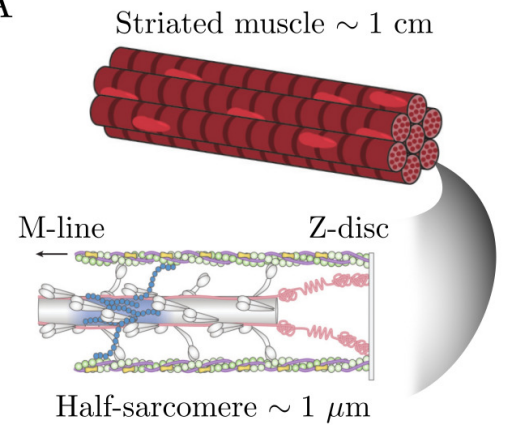

$\mathrm{C}$

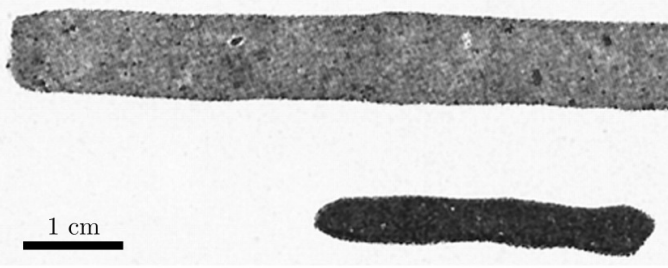

$\mathrm{B}$

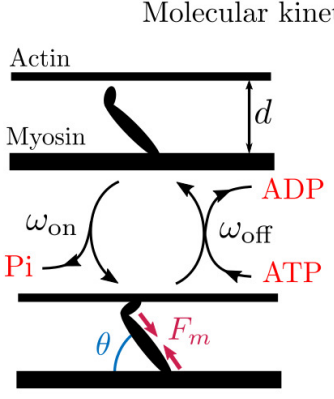

Multiscale model of muscle

Cellular active mechanics \& hydraulics

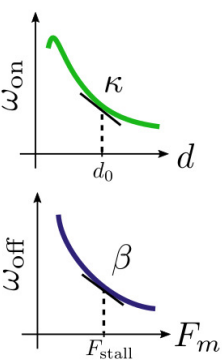

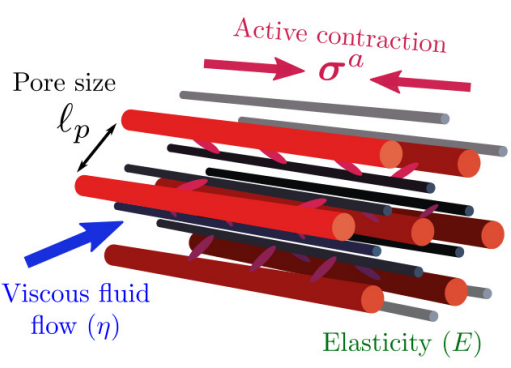

FIG. 1. Muscle is soft, wet, active and spatiotemporally multiscale. (A) Striated muscle consists of long, multinucleated cells that span several spatial scales, ranging from the contractile machinery arranged in periodically repeating sarcomeres $(\sim 1 \mu \mathrm{m})$ to the macroscopic fiber that can span 1-10 cm [8]. (B) A multiscale mathematical model for muscle (Eqs. 1-5). On the molecular scale, motor heads along the myosin thick filament stochastically bind to the actin thin filament and generate a contractile force $\left(F_{m}\right)$ upon hydrolyzing ATP, with both radial and axial components for a finite crossbridge binding angle $(\theta)$. The kinetic rates $\left(\omega_{\text {on } / \text { off }}\right)$ decrease with increasing inter-filament spacing and load on the motor, with $\kappa, \beta>0$ measuring the linearized feedback response at resting, stall conditions (see SI). On the mesoscale, the myofilament lattice is an anisotropic elastic network with a characteristic shear modulus $E$ and pore size $\ell_{p}$, that is permeated by a fluid of viscosity $\eta$, and contracts under the action of a molecularly generated active stress $\left(\boldsymbol{\sigma}^{a}\right)$. (C) Actomyosin threads contract by expelling water, changing both shape and size when placed in boiled muscle juice (a source of ATP) [11]. (D) In-vivo contraction of an electrically stimulated skeletal muscle (murine gastrocnemius) displaying spatial strain heterogeneities (right: heatmap of the 2D areal strain) measured using the stained nuclei as fiducial markers (green: undeformed, red: deformed) [18].

spatiotemporal heterogeneities in strain.

Given the empirical evidence for volumetric deformations in muscle, how can we build a model that captures and explains these effects? Very recently, studies have begun addressing this question by focusing on intramuscular pressure [26] and fluid flow within muscle microstructure $[24,27]$, but largely ignore the multiscale, elastic, active and spatial aspects of the problem. A common framework to describe sponge-like materials is poroelasticity that was initially developed to address the settling of water-logged soils [28] and other geophysical problems [29], but has since been applied to describe cartilage [30], passive muscle [31], living cells [32-34], rapid plant movements $[35,36]$, active gels [37-40] etc. Here we generalize this framework by integrating the molecular actomyosin kinetics with the anisotropic elasticity, activity and flow in the cell to describe muscle as an active self-squeezing sponge (Fig. 1B).

At a minimal level, we model the muscle cell as a cylindrical fiber of a biphasic mixture of an active porous solid ( $\phi$ : solid fraction) immersed in fluid $(1-\phi$ : fluid fraction). Assuming that $\hat{\mathbf{z}}$ is along the long-axis of the fiber, the crystalline arrangement of interdigitated filaments and flexible proteins endows the sarcomere with a uniaxially anisotropic elastic stress $\left(\boldsymbol{\sigma}^{e l}\right)$ that is linearly related to the strain tensor $\boldsymbol{\epsilon}=\left[\boldsymbol{\nabla} \mathbf{u}+(\boldsymbol{\nabla u})^{T}\right] / 2$ (displacement $\mathbf{u}=u_{r} \hat{\mathbf{r}}+u_{z} \hat{\mathbf{z}}$, assuming axisymmetry), where the drained elastic moduli are computed as a function of $\phi$ using standard homogenization techniques (see SI). In addition, the interaction between the thick (myosin) and thin (actin) filaments also leads to an active stress $\left(\boldsymbol{\sigma}^{a}\right)$. We assume that the passive elastic response of the porous solid is both anisotropic and compressible, approaching the incompressible limit only as $\phi \rightarrow 1$. The fluid stress, on the other hand, is dominated on large scales by an isotropic pressure $p$, whose gradients drive a flow velocity $(\mathbf{v})$ with viscous dissipation being consequential only on the scale of the hydraulic pore size $\ell_{p} \sim 20-55 \mathrm{~nm}[8,14]$ (SI). Mass and momentum conservation then collectively dictate overall force balance, global incompressibility and force balance in the fluid (Darcy's law) as follows (SI)

$$
\begin{aligned}
& \boldsymbol{\nabla} \cdot\left[\phi\left(\boldsymbol{\sigma}^{a}+\boldsymbol{\sigma}^{e l}\right)-(1-\phi) p \mathbf{1}\right]=\mathbf{0} \\
& \boldsymbol{\nabla} \cdot\left[\phi \partial_{t} \mathbf{u}+(1-\phi) \mathbf{v}\right]=0, \\
& (1-\phi)\left(\mathbf{v}-\partial_{t} \mathbf{u}\right)=-\frac{\ell_{p}^{2}}{\eta} \mathbf{K} \cdot \boldsymbol{\nabla} p
\end{aligned}
$$

where $\eta$ is the fluid viscosity and $\mathbf{K}(\phi)=K_{\|}(\phi) \hat{\mathbf{z}} \hat{\mathbf{z}}+$ 
bioRxiv preprint doi: https://doi.org/10.1101/2022.02.20.481216; this version posted February 20, 2022. The copyright holder for this

\section{In-vitro}

A Glycerinated skeletal muscle - Low ATP

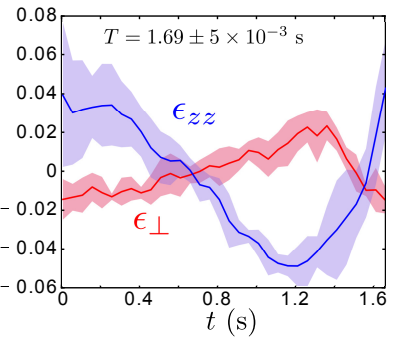

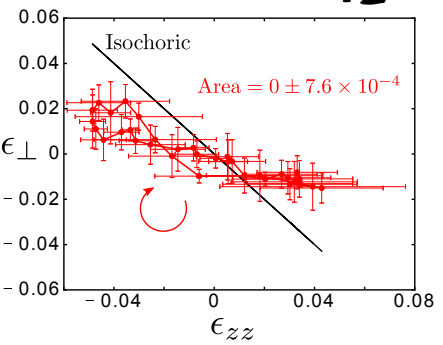

In-vivo

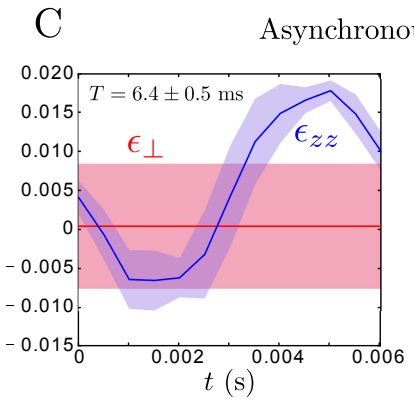

$\mathrm{B}$

Glycerinated skeletal muscle - High ATP
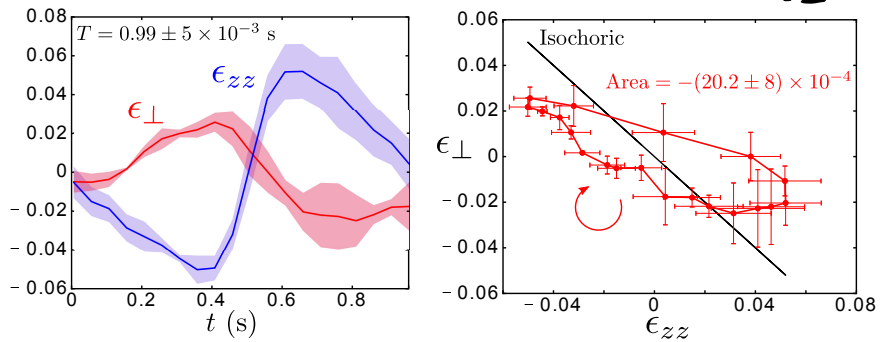

Ex-vivo

D Synchronous flight muscle
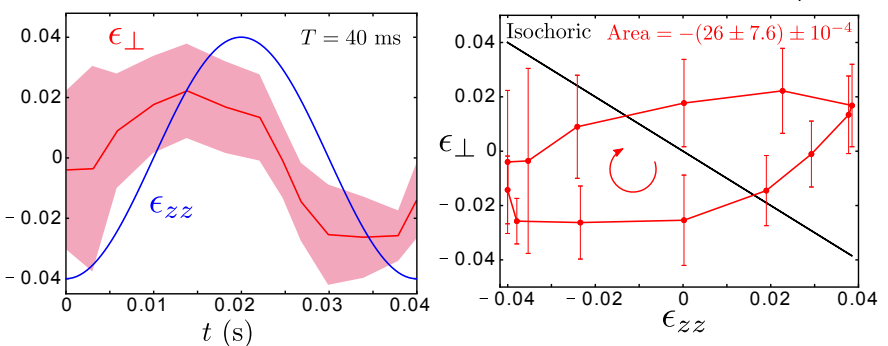

FIG. 2. Volume changes and strain cycles are generic in self-oscillating muscle. (A-B) Skinned rabbit psoas muscle fibers exhibit spontaneous sarcomeric oscillations in-vitro under different ATP concentrations and sarcomere resting lengths $[19,20]$. Deformations are generically not isochoric $\left(\epsilon_{z z}+\epsilon_{\perp}=0\right.$, black line), as a result, fluid flow is inevitable. At low ATP concentrations $(\mathrm{A})$, the axial $\left(\epsilon_{z z}\right)$ and transverse strains $\left(\epsilon_{\perp}\right)$ oscillate nearly antiphase with frequency $\omega \approx 0.6 \mathrm{~Hz}[20]$, while at higher ATP concentrations $(B)$, faster oscillations $(\omega \approx 1 \mathrm{~Hz})$ develop a strain cycle [19] that encloses a significant (signed) area, see SI. The direction of circling the loop is shown by the red arrow. (C) In-vivo measurements of strain in asynchronous flight muscle (DLM: dorsal longitudinal) of a fruit fly in tethered flight $(\omega \approx 156 \mathrm{~Hz})$ [21-23]. Axial strains plotted are obtained from high-speed optical measurements of muscle length [21] and transverse strains (computed from timeresolved X-ray diffractometry measurements with subnanometer precision, see SI) remain negligible at all times [22]. (D) Ex-vivo measurements of strain in intact synchronous flight muscle (DLM) from Manduca sexta (hawkmoth) [24], displaying nonisochoric strain cycles. The isolated, whole muscle is subject to oscillatory stretch ( $4 \%$ axial strain, $\omega=25 \mathrm{~Hz})$ and electrical stimulation (activation phase of 0.5), matching physiological in-vivo conditions. The transverse strain is estimated from time-resolved X-ray diffraction measurements of the lattice spacing. The shaded regions and error bars in A-C denote one standard deviation from averaging over multiple periods, and a $95 \%$ confidence interval in D. See SI for further details of the analysis.

$K_{\perp}(\phi)(\mathbf{1}-\hat{\mathbf{z}} \hat{\mathbf{z}})$ is a $\phi$-dependent anisotropic permeability tensor (SI). We note that the anisotropic structure of the sarcomere ensures that the lattice becomes radially impermeable to fluid flow $\left(K_{\perp} \rightarrow 0\right.$ as $\phi \rightarrow \phi_{*} \approx 0.91$, see SI) before becoming incompressible $(\phi \rightarrow 1)$. Hence volumetric deformations leading to intracellular flow are inevitable for all physiologically relevant $\phi \sim 0.1-0.22$ $[8,14](\mathrm{SI})$.

To determine the active stress $\boldsymbol{\sigma}^{a}$ due to the molecular kinetics of the force-generating myosin motors, we use a simple two state model with $n_{m}(\mathbf{x}, t)$ being the coarsegrained fraction of bound myosin motors and $\langle y\rangle(\mathbf{x}, t)$ being the average extension of the motor head, at a given time $t$ and position $\mathbf{x}$ in the cell. In the mean-field limit, the coupled dynamics of the actomyosin crossbridges is then given by (SI)

$$
\begin{aligned}
\partial_{t} n_{m}= & \omega_{\mathrm{on}}\left(\epsilon_{\perp}\right)\left(1-n_{m}\right)-\omega_{\mathrm{off}}(\langle y\rangle) n_{m}, \\
\partial_{t}\langle y\rangle= & y_{0}\left(\lambda_{\|} \partial_{t} \epsilon_{z z}+\lambda_{\perp} \partial_{t} \epsilon_{\perp}\right) \\
& -\omega_{\mathrm{on}}\left(\epsilon_{\perp}\right)\left(\frac{1-n_{m}}{n_{m}}\right)\left(\langle y\rangle-y_{0}\right),
\end{aligned}
$$

where $y_{0} \simeq 8-10 \mathrm{~nm}$ [41] is the motor prestrain generated during the powerstroke and $\lambda_{\|}, \lambda_{\perp}$ are factors associated with the geometry of the binding crossbridge $[42,43]$ (see SI). The kinetic rates minimally incorporate biophysical feedback (Fig. 1B; see SI for details) through a load-dependent unbinding rate $\left(\omega_{\text {off }}(\langle y\rangle)\right)$ capturing the myosin "catch-bond" [44] which allows for stretch-activation [45], and a strain or lattice-spacing dependent binding rate $\left(\omega_{\text {on }}\left(\epsilon_{\perp}\right)\right)$ due to mechanisms including lattice geometry [46-48], titin [49] etc., that allow 
bioRxiv preprint doi: https://doi.org/10.1101/2022.02.20.481216; this version posted February 20, 2022. The copyright holder for this preprint (which was not certified by peer review) is the author/funder. All rights reserved. No reuse allowed without permission.

A

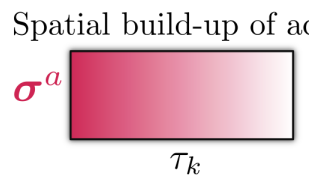

Active hydraulic oscillations

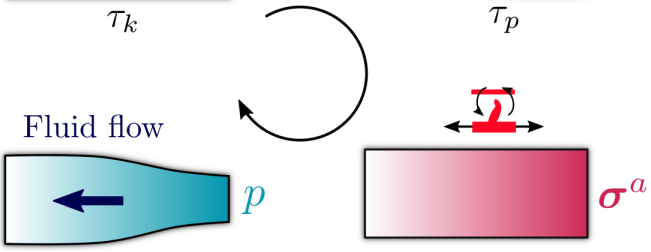

Stretch-activated myosin

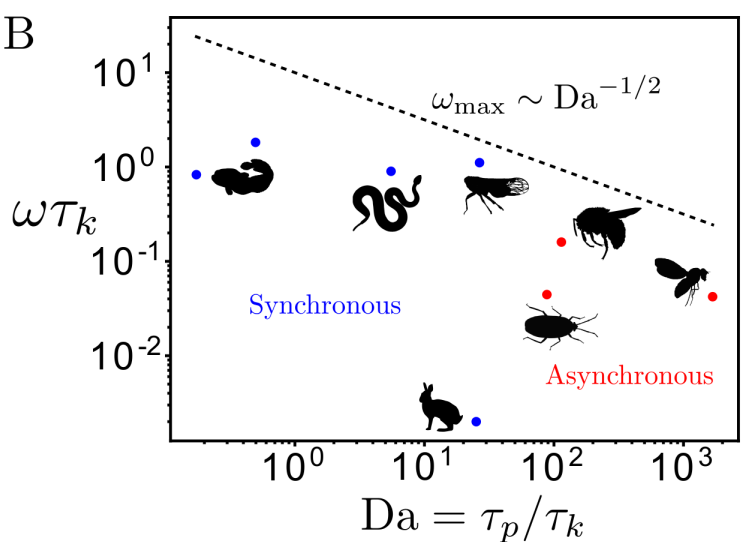

$\mathrm{C}$

Nonreciprocal response \& odd-strain cycles
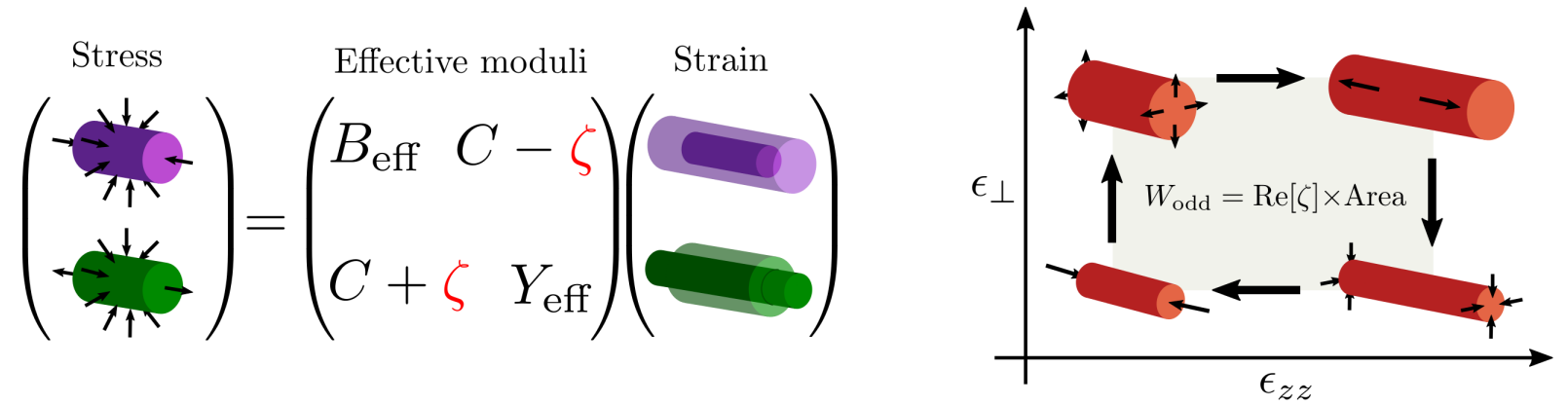

FIG. 3. Dynamic, mechanical and energetic consequences of the model. (A) Active hydraulic oscillations involving a periodic buildup of spatial gradients in active stresses along with a sloshing of fluid occur generically with a frequency $\omega \sim 1 / \sqrt{\tau_{k} \tau_{p}}$ that combines the fast crossbridge cycling time $\left(\tau_{k}\right)$ with the slow fluid permeation time $\left(\tau_{p}\right)$. (B) Characteristic oscillation frequencies $(\omega)$ of contractions in fast muscles, both synchronous (blue) and asynchronous (red) across species plotted against the estimated Damköhler number (Da, Eq. 6), with the theoretical scaling limit $\omega_{\max }$ from hydraulic oscillations plotted (dashed line, Eq. 7); see SI for details. Consistent with our prediction, the rates of spontaneous muscular contractions in the representative examples do not exceed the limit set by active hydraulics. (C) A pictorial representation of a stress-strain relation involving shear and volume changes. As muscle is active, the matrix of effective moduli can be nonsymmetric, with the asymmetry quantified by an odd or nonreciprocal modulus $\zeta$. While the bulk $\left(B_{\text {eff }}\right)$ and Young's $\left(Y_{\text {eff }}\right)$ moduli are present in isotropic solids, the passive coupling $C$ is only present in anisotropic solids and $\zeta$ requires both anisotropy and activity to be present. The presence of $\zeta \neq 0$ allows work to be actively produced by cycling strains in different directions, say axial and radial (right). The work done by the odd-modulus ( $W_{\text {odd }}$, Eq. 8$)$ is proportional to odd-elasticity $(\operatorname{Re}[\zeta])$ and the signed area enclosed by the closed loop in strain space.

for length-based regulation of force underlying the wellknown Frank-Starling law [4]. Assuming a motor stiffness $k_{m}$, motor size $d_{m}$ and a linear density of motor heads $N$ along a thick filament, the active contractile force (per motor) is $F_{m}=-k_{m} n_{m}\langle y\rangle$ which gives rise to an anisotropic active stress $\boldsymbol{\sigma}^{a}=-N d_{m}\left[F_{\|} \hat{\mathbf{z}} \hat{\mathbf{z}}+F_{\perp}(\mathbf{1}-\hat{\mathbf{z}} \hat{\mathbf{z}})\right]$ [50] where $F_{\|}=F_{m} \cos \theta\left(F_{\perp}=F_{m} \sin \theta\right)$ is the longitudinal (transverse) active force governed by the crossbridge binding angle $\theta[42,43,47]$ (Fig. 1B). Eqs. 1-5 supplemented by appropriate boundary and initial conditions complete the specification of our multiscale continuum model.

To understand the consequences of the model, we consider two simple limits. In the passive, isotropic limit $\left(\boldsymbol{\sigma}^{a}=\mathbf{0}, K_{\|}=K_{\perp}\right)$, we recover the classical result [28] that the hydrostatic pressure $(p)$ equilibrates across a length $L$ diffusively on a poroelastic timescale $\tau_{p} \sim(\eta / E)\left(L / \ell_{p}\right)^{2}(\mathrm{SI})$, that combines the material (viscosity $\eta$, elastic modulus $E$ ) with the microstructural (pore size $\ell_{p}$ ). In contrast, in the active case, upon neglecting spatial heterogeneities and fluid flow $(\boldsymbol{\nabla} p \simeq \mathbf{0})$, our model matches previous kinetic theories of molecular motor assemblies [51-54], where the kinetic timescale, $\tau_{k}=\left[\omega_{\text {on }}+\omega_{\text {off }}\left(y_{0}\right)\right]^{-1}(\mathrm{SI})$ controls the residence time of bound motors and the rate of buildup of active stress. In this situation, if the load-dependent feedback is strong enough $\left(y_{0} \tau_{k} \omega_{\text {off }}^{\prime}\left(y_{0}\right)>1, \mathrm{SI}\right)$, the molecular reaction develops an oscillatory instability with a characteristic frequency $\omega \sim 1 / \tau_{k}$ (SI). Combining the two limits, a key dimensionless parameter emerges - the poroelastic Damköhler number

$$
\mathrm{Da}=\frac{\tau_{p}}{\tau_{k}},
$$


that captures the relative importance of fluid permeation (a mesoscopic time) to actomyosin kinetics (a molecular time). Assuming typical values for $E \sim 0.1-1 \mathrm{MPa}$, $\eta \sim 10^{-2}$ Pa.s, $\ell_{p} \sim 20 \mathrm{~nm}, \tau_{k} \sim 1-10 \mathrm{~ms}$ and $L \sim$ $10-100 \mu \mathrm{m}[14,55,56]$, we obtain a wide range of Da $\sim$ $0.1-10^{3}$ that is accessible to and seems to be exploited by the evolutionary range of muscle physiology (Fig. 3B, see SI).

When poroelastic, active and kinetic effects are all incorporated, we obtain a novel oscillatory instability that relies on spatial gradients in strain (Fig. 3A). A local buildup of active stress (on timescale $\tau_{k}$ ) squeezes the sarcomere, forcing fluid to flow and distend neighbouring regions of the lattice (on a timescale $\tau_{p}$ ), which in turn induces further buildup of myosin via stretch activation (in space). To see this, we consider a minimal one-dimensional (1D) description where axial force balance along the muscle fiber implies that $\partial_{z}\left[\phi\left(\sigma_{z z}^{a}+\right.\right.$ $\left.\left.E \epsilon_{z z}\right)-(1-\phi) p\right]=0$ (assuming $\epsilon_{\perp} \approx 0$ ), while poroelastic flow dictates $\partial_{t} \epsilon_{z z}=K_{\|}\left(\ell_{p}^{2} / \eta\right) \partial_{z}^{2} p$. Considering the slowest modes on scale of the system $(L)$, these equations together (see SI) yield $\tau_{p} \partial_{t} \epsilon_{z z} \sim-\left[\epsilon_{z z}+\sigma_{z z}^{a} / E\right]$. In the limit when the active stress is slaved to the density of bound motors (see SI), we can write $\sigma_{z z}^{a} \sim$ $\alpha_{\|} E n_{m}\left(\alpha_{\|}>0\right.$ is a nondimensional contractility, SI $)$ and linearize the kinetics about the steady state motor density $\left(n_{m}=n_{m}^{0}+\delta n_{m}, n_{m}^{0}=\omega_{\text {on }} \tau_{k}\right)$ to obtain $\partial_{t} \delta n_{m}=-\delta n_{m} / \tau_{k}-\beta \lambda_{\|} n_{m}^{0} \partial_{t} \epsilon_{z z}$ that includes the (catch-bond driven) positive feedback mechanism through $\beta=y_{0} \omega_{\text {off }}^{\prime}\left(y_{0}\right) / \omega_{\text {off }}\left(y_{0}\right)>0$ (SI). For strong enough activity $\left(\alpha_{\|} \gtrsim \mathrm{Da} / \beta \lambda_{\|} n_{m}^{0}\right.$, SI $)$, the coupled dynamics undergoes a Hopf bifurcation resulting in the spontaneous emergence of active hydraulic oscillations with a scaled characteristic frequency

$$
\omega \tau_{k} \sim \frac{1}{\sqrt{\mathrm{Da}}},
$$

that involves both the kinetic and the poroelastic time scales; a careful calculation shows that this phenomenon persists more generally (see SI). Strikingly, any spatiotemporal volumetric deformation (inevitable at finite Da) forces hydraulic oscillations, even when the instability mechanism is kinetic (see SI), hence offering a natural explanation for the experimental data in Fig. 2. Thus, we see that active hydraulics, rather than just kinetics, determines the fastest rate of spontaneous muscle contraction; in Fig. 3B, we plot the scaling relation in Eq. 7 and compare it to existing experimental data on muscular contractions across the animal kingdom. Using representative estimates of the poroelastic, kinetic and contraction time scales in fast sonic, flight and skeletal muscles (see SI for details), we find that the data naturally clusters synchronous (blue) and asynchronous (red) muscles separately and is consistent with the maximal contraction rate being set by active hydraulics.

Beyond dynamics, our model also exhibits unusual mechanics. For small deformations, we can linearize about the resting state, Fourier transform in time $(\boldsymbol{\epsilon}(\omega)=$ $\left.\int \mathrm{d} t e^{-i \omega t} \boldsymbol{\epsilon}(t)\right)$ and cast our dynamical equations (Eqs. 4$3)$ as an effective (visco)elastic constitutive relation $\sigma_{i j}(\omega)=\mathcal{A}_{i j k l}(\omega) \epsilon_{k l}(\omega)$ that linearly relates the total stress $\left(\boldsymbol{\sigma}=\phi\left(\boldsymbol{\sigma}^{e l}+\boldsymbol{\sigma}^{a}\right)-(1-\phi) p \mathbf{1}\right)$ to the strain tensor, shown pictorially for the shear and isotropic components in Fig. 3C (see SI for details). The complex modulus $\mathcal{A}$ includes both an (in-phase) elastic response $\operatorname{Re}[\mathcal{A}]$ and an (out-of-phase) viscous response $\operatorname{Im}[\mathcal{A}]$. In a passive system, time-reversal symmetry enforces $\mathcal{A}_{i j k l}(\omega)=\mathcal{A}_{k l i j}(\omega)$, but in an active system such as muscle, the lack of energy conservation allows new nonsymmetric terms (recently christened 'odd (visco)elasticity' $[60,61]$ in chiral active media) that violate a fundamental property of mechanics - Maxwell-Betti reciprocity [62]. Thus, in addition to the bulk $\left(B_{\text {eff }}(\omega)\right)$ and Young's moduli $\left(Y_{\text {eff }}(\omega)\right)$ that govern the standard compressive and shear response and acquire frequency dependent corrections from motor kinetics (SI), muscle's uniaxial anisotropy couples isotropic dilations to shear via a passive anisotropic modulus $C(\omega)$ along with an exclusively active and nonreciprocal 'odd-modulus' $\zeta(\omega)$ (Fig. 3C, left; see SI). Note, spatial anisotropy and activity are sufficient for odd (visco)elasticty to emerge in muscle, even without chirality. Interestingly, the possible presence of such an odd-modulus in muscle was noted in passing in old work [63], though its effects were unrecognized and forgotten.

A unique consequence of nonreciprocal mechanics is the ability to generate work from cycles of strain (Fig. 3C, right). Upon writing $\mathcal{A}_{i j k l}=\mathcal{A}_{i j k l}^{e}+\mathcal{A}_{i j k l}^{o}$ in terms of 'even' and 'odd' tensors (under the exchange of $i j$ and $k l$ indices), we can compute the cumulative mechanical work in a cycle as $W=-\oint \sigma_{i j} \mathrm{~d} \epsilon_{i j}(W>$ 0 when work is produced, $W<0$ when dissipated), which includes two qualitatively different terms $W=$ $W_{\text {even }}+W_{\text {odd }}(\mathrm{SI}) ;$ an even viscous term $W_{\text {even }}=$ $-\oint \mathrm{d} t\left(\operatorname{Im}\left[\mathcal{A}_{i j k l}^{e}\right] / \omega\right) \dot{\epsilon}_{i j} \dot{\epsilon}_{k l}$ that depends on the traversal frequency $\omega$ and strain rates $(\dot{\boldsymbol{\epsilon}})$ and an odd elastic term $W_{\text {odd }}=\oint \mathrm{d} t \operatorname{Re}\left[\mathcal{A}_{i j k l}^{o}\right] \epsilon_{k l} \mathrm{~d} \epsilon_{i j}$ that depends on strain (see SI). While $W_{\text {even }}$ is simply an anisotropic generalization of standard viscous dissipation $(\operatorname{Im}[\mathcal{A}] / \omega$ is like a viscosity), an intuitive explanation for $W_{\text {odd }}$ is that, in the absence of energy conservation, cyclic deformations in different directions do not bring the system back to its initial energy, hence work can be either produced or absorbed. In the axisymmetric limit, relevant for muscle, we can simplify $W_{\text {odd }}$ as (SI)

$$
W_{\text {odd }}=\frac{\operatorname{Re}[\zeta]}{2} \oint\left[\epsilon_{z z} \mathrm{~d} \epsilon_{\perp}-\epsilon_{\perp} \mathrm{d} \epsilon_{z z}\right],
$$

i.e., work from odd elasticity depends on the oddmodulus $\zeta$ and the area enclosed by a loop in the space of axial and transverse strains (Fig. 3C, right). Crucially this mechanism of power generation relies on threedimensional (3D) spatial deformations (axial and radial), rather than temporal variations.

Where might we see these effects in muscle? While a fully $3 \mathrm{D}$ characterization of muscle's viscoelastic response 

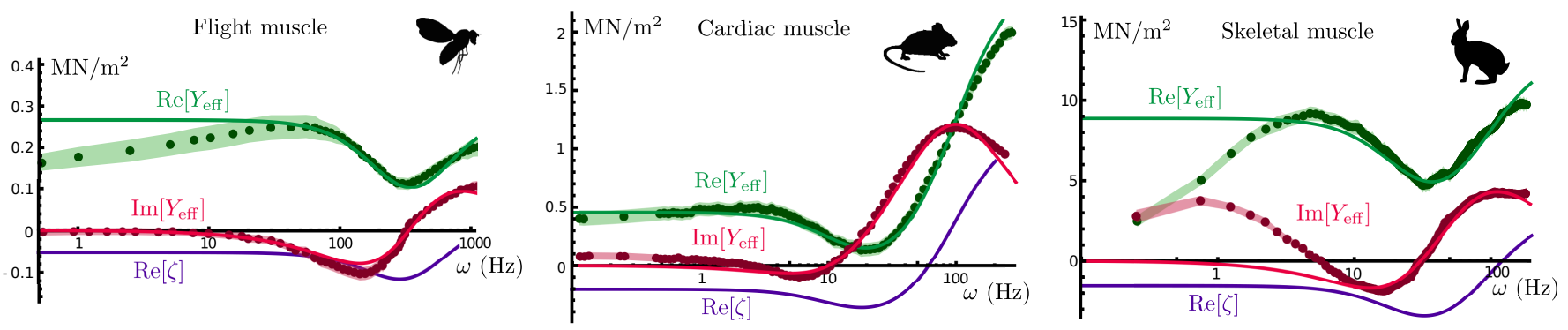

FIG. 4. Active and nonreciprocal viscoelasticity across muscle types and species. Model fits of linear response (lines) to experimental data (circles, shaded region is one standard deviation) of sinusoidal uniaxial rheology compiled from Ref. [57] (Drosophila flight muscle), Ref. [58] (mouse cardiac muscle) and Ref. [59] (rabbit skeletal muscle). As the muscle fibers are small and permeabilized, we assume affine deformations and neglect pressure gradients (see SI for fit details). The real and imaginary parts of the response correspond to the elastic (Re $\left[Y_{\text {eff }}\right]$, green) and the viscous $\left(\operatorname{Im}\left[Y_{\text {eff }}\right]\right.$, magenta) moduli. The low frequency response in the experiments sometimes includes a weak power law or logarithmic dependence on frequency, possibly arising from internal passive processes that we neglect. Estimates of the nonreciprocal or odd elastic modulus Re[ $\zeta]$ are shown in blue (see SI for details).

is currently unavailable, we use our model to analyze existing muscle rheology experiments [57-59] in a simpler $1 \mathrm{D}$ geometry that measures the uniaxial response $\left(\sigma_{z z}\right)$ in skinned muscle fibers subject to small amplitude oscillatory axial strains $\left(\epsilon_{z z}\right)$. By compiling data across muscle types and species (Drosophila insect flight [57], mouse cardiac [58] and rabbit skeletal [59]), we fit our biophysical model to the measured effective (complex) Young's modulus $Y_{\text {eff }}(\omega)=\sigma_{z z}(\omega) / \epsilon_{z z}(\omega)$ (Fig. 4, see SI for analysis details).

In all three cases, a common characteristic response is seen - the elastic modulus ( $\operatorname{Re}\left[Y_{\text {eff }}\right]$, Fig. 4 green) transitions from a low frequency (largely passive) stiffness to a high-frequency rigor-like response from transiently crosslinked actomyosin, passing through an intermediary softening on time scales associated with crossbridge cycling, i.e., when the kinetic cycling of motors enhances compliance via filament sliding. The viscous modulus ( $\operatorname{Im}\left[Y_{\text {eff }}\right]$, Fig. 4 magenta) on the other hand is negative at low frequency (hence active and work producing), switching to positive (dissipative) values at higher frequencies (skeletal muscle displays additional features at low frequency arising from passive dissipation in sarcomeric polymers that we neglect, Fig. 4 right). In the $1 \mathrm{D}$ setting, the negative viscous modulus offers the only route to produce positive work through temporal changes in strain and odd-elastic effects are absent. Nonetheless, we can use our model fit and known structural parameters to estimate the frequency dependent odd-elastic modulus $(\operatorname{Re}[\zeta])$ for the various muscle types (Fig. 4, blue lines; see SI). Notably, we see that the odd-modulus is predominantly negative and it remains nonvanishing at low frequencies, where its magnitude is controlled by the active stress and the strain based feedback on myosin kinetics (SI). As Fig. 2B,D show, strain cycles (enclosing negative area) can be seen during spontaneous muscle contractions, which when combined with our model prediction of $\operatorname{Re}[\zeta]<0$ and Eq. 8 are consistent with strain cycles actively producing work through odd elasticity.

Our work demonstrates that a minimal multiscale biophysical model of muscle as an active sponge is sufficient to capture many of muscle's dynamic, mechanical and energetic properties relevant to physiology. By recognizing the importance of spatial strain gradients and the time constraint of fluid movement, we have shown that the maximum rate of muscle contraction $\omega_{\max }=\omega_{\max }\left(\tau_{k}, \ell_{p}, \eta, E, L, \cdots\right)$ is an emergent timescale of active hydraulic oscillations that combines a molecular kinetic time scale $\left(\tau_{k}\right)$ with a mesoscopic poroelastic time scale $\left(\tau_{p}\right)$ that incorporates micro-structural $\left(\ell_{p}\right)$ and macro-geometric $(L)$ scales as well as material properties $(\eta, E)$. Our analysis of published 3D spatial deformation data complements previous temporal studies of muscle rheology and highlights how muscle functions as an active elastic engine, whereby work can be produced from strain cycles via an emergent nonreciprocal response naturally present in anisotropic active solids. All together, our results suggest that a spatiotemporally integrated and multiscale view of muscle physiology is essential for an understanding of this ubiquitous effector of animal behaviour.

\section{ACKNOWLEDGMENTS}

SS acknowledges support from the Harvard Society of Fellows, and LM acknowledges partial support from the NSF Simons Center for Quantitative Biology, the Simons Foundation and the Henri Seydoux Fund. We thank Shriya Srinivasan for useful discussions. 
bioRxiv preprint doi: https://doi.org/10.1101/2022.02.20.481216; this version posted February 20, 2022. The copyright holder for this

[1] L. C. Rome and S. L. Lindstedt, Physiology 13, 261 (1998).

[2] D. A. Syme and R. K. Josephson, Integrative and comparative biology 42, 762 (2002).

[3] A. Huxley, Reflections on Muscle, Sherrington lectures (Liverpool University Press, 1980).

[4] D. M. Needham, Machina carnis: the biochemistry of muscular contraction in its historical development (Cambridge University Press, 1971).

[5] M. Nyitrai, R. Rossi, N. Adamek, M. A. Pellegrino, R. Bottinelli, and M. A. Geeves, Journal of molecular biology 355, 432 (2006).

[6] D. M. Swank, V. K. Vishnudas, and D. W. Maughan, Proceedings of the National Academy of Sciences 103, 17543 (2006).

[7] A. F. Mead, N. Osinalde, N. Ørtenblad, J. Nielsen, J. Brewer, M. Vellema, I. Adam, C. Scharff, Y. Song, U. Frandsen, et al., Elife 6, e29425 (2017).

[8] J. D. Powers, S. A. Malingen, M. Regnier, and T. L. Daniel, Annual Review of Biophysics 50, 373 (2021).

[9] B. Kaminer, The Journal of general physiology 46, 131 (1962).

[10] K. Trombitás, P. Baatsen, J. Schreuder, and G. H. Pollack, Journal of Muscle Research \& Cell Motility 14, 573 (1993).

[11] A. Szent-Györgyi, Studies from the Institute of Medical Chemistry, University of Szeged 1, 17 (1942).

[12] A. G. Szent-Györgyi, The Journal of general physiology 123, 631 (2004).

[13] G. Cecchi, M. Bagni, P. Griffiths, C. Ashley, and Y. Maeda, Science 250, 1409 (1990).

[14] B. M. Millman, Physiological reviews 78, 359 (1998).

[15] J. Pinto and R. Win, American Journal of PhysiologyHeart and Circulatory Physiology 233, H410 (1977).

[16] I. Neering, L. Quesenberry, V. Morris, and S. Taylor, Biophysical journal 59, 926 (1991).

[17] S. Ghosh, J. G. Cimino, A. K. Scott, F. W. Damen, E. H. Phillips, A. I. Veress, C. P. Neu, and C. J. Goergen, ACS biomaterials science \& engineering 3, 2798 (2017).

[18] S. Ghosh, B. Seelbinder, J. T. Henderson, R. D. Watts, A. K. Scott, A. I. Veress, and C. P. Neu, Cell reports 27, 1607 (2019).

[19] T. Washio, S. A. Shintani, H. Higuchi, S. Sugiura, and T. Hisada, Scientific reports 9, 1 (2019).

[20] F. Kono, S. Kawai, Y. Shimamoto, and S. Ishiwata, Scientific reports 10, 1 (2020).

[21] W. P. Chan and M. H. Dickinson, The Journal of experimental biology 199, 2767 (1996).

[22] T. Irving and D. Maughan, Biophysical journal 78, 2511 (2000).

[23] M. Dickinson, G. Farman, M. Frye, T. Bekyarova, D. Gore, D. Maughan, and T. Irving, Nature 433, 330 (2005)

[24] J. A. Cass, C. D. Williams, T. C. Irving, E. Lauga, S. Malingen, T. L. Daniel, and S. N. Sponberg, Biophysical Journal (2021).

[25] S. A. Malingen, A. M. Asencio, J. A. Cass, W. Ma, T. C. Irving, and T. L. Daniel, Journal of Experimental Biology 223, jeb224188 (2020).

[26] D. A. Sleboda and T. J. Roberts, Proceedings of the National Academy of Sciences 117, 1772 (2020).
[27] S. A. Malingen, K. Hood, E. Lauga, A. Hosoi, and T. L. Daniel, Archives of Biochemistry and Biophysics $\mathbf{7 0 6}$, 108923 (2021).

[28] M. A. Biot, Journal of applied physics 12, 155 (1941).

[29] H. F. Wang, Theory of linear poroelasticity with applications to geomechanics and hydrogeology (Princeton University Press, 2017).

[30] V. C. Mow, M. H. Holmes, and W. M. Lai, Journal of biomechanics 17, 377 (1984).

[31] M. Yang and L. A. Taber, Journal of biomechanics 24, 587 (1991).

[32] G. T. Charras, T. J. Mitchison, and L. Mahadevan, Journal of cell science 122, 3233 (2009).

[33] E. Moeendarbary, L. Valon, M. Fritzsche, A. R. Harris, D. A. Moulding, A. J. Thrasher, E. Stride, L. Mahadevan, and G. T. Charras, Nature materials 12, 253 (2013).

[34] A. Mogilner and A. Manhart, Annual Review of Fluid Mechanics 50, 347 (2018).

[35] Y. Forterre, J. M. Skotheim, J. Dumais, and L. Mahadevan, Nature 433, 421 (2005).

[36] J. M. Skotheim and L. Mahadevan, Science 308, 1308 (2005).

[37] A. C. Callan-Jones and F. Jülicher, New Journal of Physics 13, 093027 (2011).

[38] M. Radszuweit, S. Alonso, H. Engel, and M. Bär, Physical review letters 110, 138102 (2013).

[39] Y. Ideses, V. Erukhimovitch, R. Brand, D. Jourdain, J. S. Hernandez, U. Gabinet, S. Safran, K. Kruse, and A. Bernheim-Groswasser, Nature communications 9, 1 (2018).

[40] C. A. Weber, C. H. Rycroft, and L. Mahadevan, Physical review letters 120, 248003 (2018).

[41] A. F. Huxley and R. M. Simmons, Nature 233, 533 (1971).

[42] M. Schoenberg, Biophysical journal 30, 51 (1980).

[43] M. Schoenberg, Biophysical journal 30, 69 (1980).

[44] C. Veigel, J. E. Molloy, S. Schmitz, and J. KendrickJones, Nature cell biology 5, 980 (2003).

[45] J. W. S. Pringle, Proceedings of the Royal Society of London. Series B. Biological Sciences 201, 107 (1978).

[46] T. C. Irving, J. Konhilas, D. Perry, R. Fischetti, and P. P. De Tombe, American Journal of Physiology-Heart and Circulatory Physiology 279, H2568 (2000).

[47] C. D. Williams, M. Regnier, and T. L. Daniel, PLoS computational biology 6, e1001018 (2010).

[48] C. D. Williams, M. K. Salcedo, T. C. Irving, M. Regnier, and T. L. Daniel, Proceedings of the Royal Society B: Biological Sciences 280, 20130697 (2013).

[49] Y. Ait-Mou, K. Hsu, G. P. Farman, M. Kumar, M. L. Greaser, T. C. Irving, and P. P. de Tombe, Proceedings of the National Academy of Sciences 113, 2306 (2016).

[50] M. C. Marchetti, J.-F. Joanny, S. Ramaswamy, T. B. Liverpool, J. Prost, M. Rao, and R. A. Simha, Reviews of Modern Physics 85, 1143 (2013).

[51] F. Jülicher and J. Prost, Physical review letters 78, 4510 (1997).

[52] S. Günther and K. Kruse, new Journal of physics 9, 417 (2007).

[53] T. Guérin, J. Prost, P. Martin, and J.-F. Joanny, Current opinion in cell biology 22, 14 (2010). 
[54] T. Guérin, J. Prost, and J.-F. Joanny, The European Physical Journal E 34, 1 (2011).

[55] A. M. Gordon, E. Homsher, and M. Regnier, Physiological reviews 80, 853 (2000).

[56] R. K. Josephson, J. G. Malamud, and D. R. Stokes, Journal of Experimental Biology 203, 2713 (2000).

[57] B. C. Tanner, G. P. Farman, T. C. Irving, D. W. Maughan, B. M. Palmer, and M. S. Miller, Biophysical journal 103, 1275 (2012).

[58] B. M. Palmer, T. Suzuki, Y. Wang, W. D. Barnes, M. S. Miller, and D. W. Maughan, Biophysical journal 93, 760 (2007).
[59] M. Kawai and P. W. Brandt, Journal of Muscle Research \& Cell Motility 1, 279 (1980).

[60] C. Scheibner, A. Souslov, D. Banerjee, P. Surówka, W. T. Irvine, and V. Vitelli, Nature Physics 16, 475 (2020).

[61] D. Banerjee, V. Vitelli, F. Jülicher, and P. Surówka, Physical Review Letters 126, 138001 (2021).

[62] H. Nassar, B. Yousefzadeh, R. Fleury, M. Ruzzene, A. Alù, C. Daraio, A. N. Norris, G. Huang, and M. R. Haberman, Nature Reviews Materials 5, 667 (2020).

[63] G. I. Zahalak, Journal of theoretical biology 182, 59 (1996). 